\section{Bidirectional dynamic data transmission through a rotary interface}

\author{
Dagong Jia \\ Wencai Jing \\ Yimo Zhang \\ Guanghui Wang \\ Feng Tang \\ Jin Zhang \\ Tianjin University \\ College of Precision Instrument and \\ Opto-electronics Engineering \\ Key Laboratory of Opto-electronics \\ Information and Technical Science \\ Ministry of Education \\ Tianjing 300072, China
}

\begin{abstract}
A multichannel bidirectional dynamic data transmission system (DDTS) through a rotary interface with one fiber is designed based on a fabricated single pass fiber optic rotary joint. The feasibility of transmission system is tested at both 1310- and 1550-nm wavelength bands. The performance of this DDTS was measured using optical spectrum analyzer and lightwave multimeter. The insertion losses of DDTS were 1.55 and $1.20 \mathrm{~dB}$ at 1310- and 1550-nm wavelength bands, respectively. The total bandwidth of the DDTS is more than $170 \mathrm{~nm}$. (c) 2005 Society of Photo-Optical Instrumentation Engineers. [DOI: 10.1117/1.1906235]
\end{abstract}

Subject terms: dynamic data transmission; fiber optic rotary joints; wavelength division multiplexing; insertion losses; rotary interfaces.

Paper L040867 received Nov. 25, 2004; revised manuscript received Dec. 21, 2004; accepted for publication Feb. 16, 2005; appeared online Feb. 18, 2005; published online May 11, 2005.

With the rapid development of communication, a highspeed, rotating-to-fixed dynamic data transmission system (DDTS) is needed in some fields. Usually, a fiber optic rotary joint (FORJ) can be used to transmit both analog and digital signals through a rotating interface. Compared with electrical slip rings, FORJ is immune to electromagnetic interferences, ${ }^{1}$ but has the disadvantage of high manufacturing and assembly cost.

To ensure that the optical signal is efficiently transmitted, both ends of the single-mode fiber are required to be held very closely and in proximity so that a minimal amount of light is lost in the gap. ${ }^{2}$ In this letter, a singlechannel FORJ was designed using C-lenses, the working principle and structure of which are similar to those of graded-refracted-index (GRIN) lenses. ${ }^{3}$ Compared with GRIN lenses, the insertion loss of C-lenses is better when the working distance is longer than $50 \mathrm{~mm}^{4}$ Through precise mechanical design, the insertion loss of single channel FORJ is less than $2 \mathrm{~dB}$ at either the $1310-$ or $1550-\mathrm{nm}$ wavelength. To enhance the capability of the FORJ, a

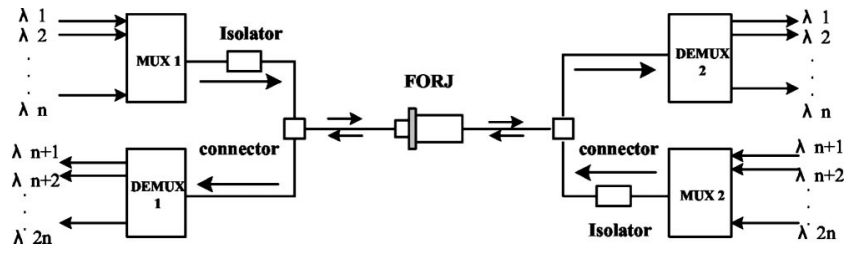

E

Fig. 1 Principle of dynamic data transmission system.

wavelength division multiplexing (WDM) technique was adopted to increase the signal transmission capacity. ${ }^{5}$

The working principle of DDTS is shown in Fig. 1. The wavelengths $\lambda_{1}, \lambda_{2}, \ldots, \lambda_{n}$ are first sent to WDM multiplexer 1 (MUX 1) where the input signals are combined into an optical single-mode (SM) fiber. Then they are sent to an isolator where the input signals are isolated from the output signals from the FORJ to reduce the noise of the dynamic transmission system. The optical signals arrive at the FORJ through the connector and are transferred across a rotating interface. Finally the received light is demultiplexed and sent into the output channels. In this way, multichannel signals from a rotary platform can be transferred to the stationary platform. At the same time, the wavelengths $\lambda_{n+1}, \lambda_{n+2}, \ldots, \lambda_{2 n}$ at the other end of DDTS are transmitted to MUX 2 and the input optical signals arrive at the FORJ. Then the input optical signals pass the rotating interface through the optical isolator and connector. Finally the $\lambda_{n+1}, \lambda_{n+2}, \ldots, \lambda_{2 n}$ are transmitted into the output channels by DEMUX 1. Because the optical components including the FORJ are all bidirectionally operable and passive components, the DDTS supports bidirectional optical transmission as well as a data transfer rate of hundreds of Gbps.

To test the feasibility of the multichannel bidirectional DDTS, an optical signal transmission system on both 1310and 1550-nm wavelengths was designed and fabricated. A $1310 / 1550-n m$ WDM is used as the wavelength MUX and DEMUX. A 1550- and 1310-nm laser diode (LD) serve as the input light source. The 1310- and 1550-nm operating wavelengths are sent into the DEMUX/MUX in two oppo-

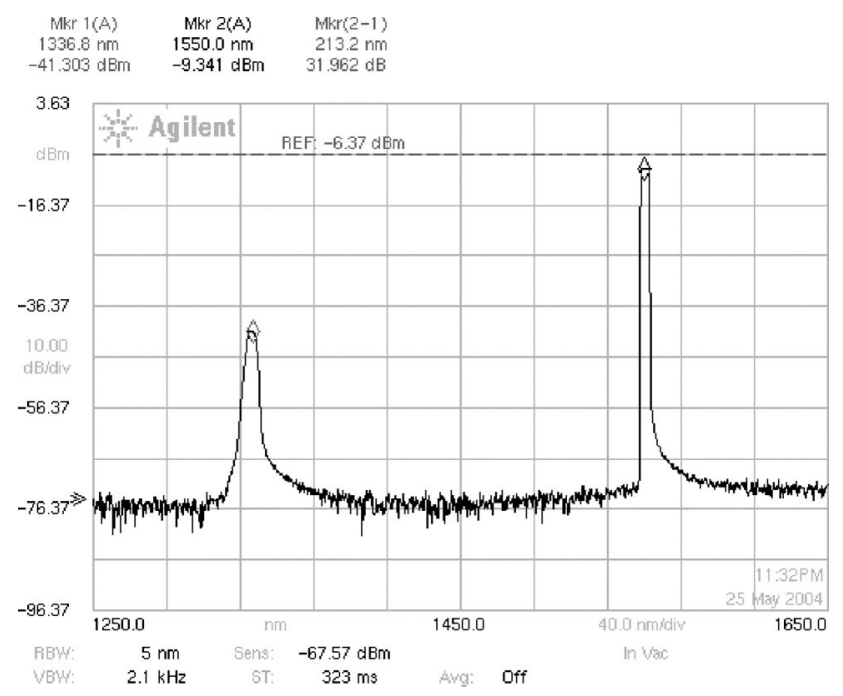

Fig. 2 Output 1 spectrum of DDTS. 


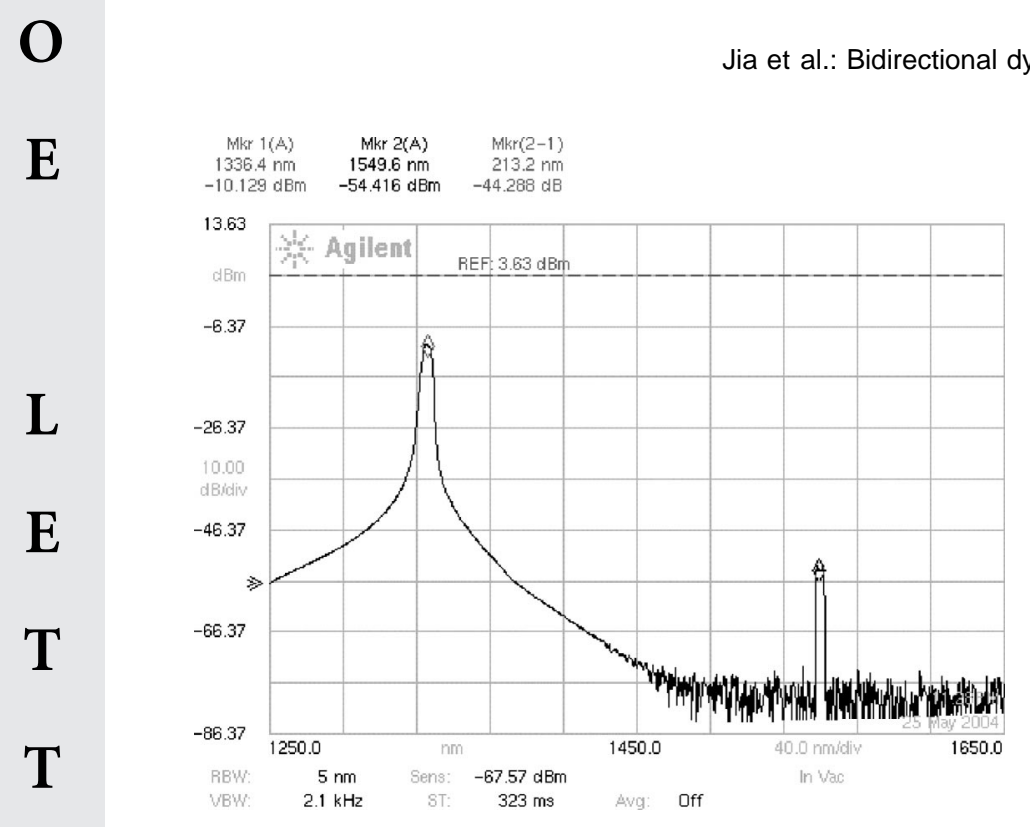

$\mathbf{E}$

Fig. 3 Output 2 spectrum of FORJ.

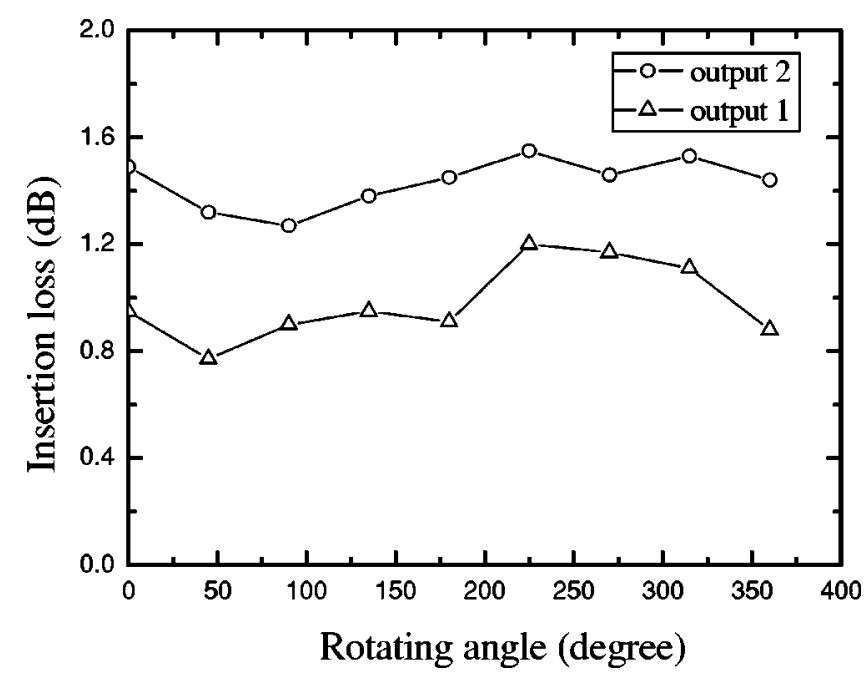

Fig. 4 Relationship between the insertion loss of DDTS and its rotation angle.

To summarize, a multichannel bidirectional DDTS using WDM technique is designed based on a single-channel FORJ. The feasibility of this DDTS was tested at both the 1310- and 1550-nm wavelength bands. The performance of this DDTS was monitored using OSA and LM. Across the rotating interface, it can bidirectionally transmit a large number of dynamic optical signals with insertion loss around $1.55 \mathrm{~dB}$ at the $1310-\mathrm{nm}$ wavelength band and 1.20 $\mathrm{dB}$ at the 1550-nm wavelength band. The performances of this DDTS using WDM technique meet the bandwidth requirements of optical fiber communication systems.

\section{Acknowledgments}

This work was supported by the National Natural Science Fund of China under contact No. 60377031 and the Natural Science Fund of Tianjin under grant No. 023602411.

\section{References}

1. W. Jing, Y. Zhang, G. Zhou, D. Jia, and B. Liu, "Design of single channel optical rotary joint using bulk optical detector," Opt. Eng. 42(11), 3285-3289 (2003).

2. G. F. Dorsey, "Fiber optic rotary joints-a review," Proc. IEEE 5, 37-41 (1982).

3. Y. C. Shi, L. Klafter, and E. E. Harstead, "A dual-fiber optical rotary joint," J. Lightwave Technol. LT-3(5), 999-1004 (1985).

4. W. Suqin, R. Yu, Y. Dongliang et al., "The calculation and analyzing of the RL of C-lens collimator," Opto. Tech. Inf. 16(1), 24-28 (2003) (in Chinese).

5. J. Dagong, Z. Yimo, J. Wencai et al., "Design of passive multi-pass fiber optic rotary joint," J. Tianjing Univ. 37(5), 382-385 (2004) (in Chinese).

6. W. W. Koth and S. K. Nauman, "Proof-of-concept model of a multichannel off-axis passive bidirectional fiber optic rotary joint," Proc. SPIE 931, 94-97 (1988).

7. N. Ito and T. Numazaki, "Optical two-way communication system using a rotary coupler," Appl. Opt. 24(14), 2221-2223 (1985). 